\title{
Article \\ Global Well-Posedness for the fractional Navier-Stokes-Coriolis equations in Function spaces Characterized by Semigroups
}

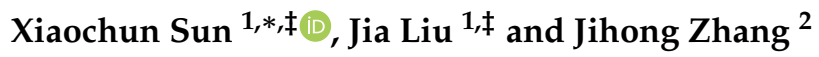 \\ 1 College of Mathematics and Statistics, Northwest Normal University, Lanzhou 730070, PR China; \\ sunxc@mail.bnu.edu.cn \\ 2 School of Mathematics, Lanzhou City University, Lanzhou 730070, PR China; zhjhzhangjihong@163.com \\ * Correspondence: sunxc@mail.bnu.edu.cn; Tel.:+86-1889-318-3008 \\ $\ddagger \quad$ These authors contributed equally to this work.
}

\begin{abstract}
We studies the initial value problem for the fractional Navier-Stokes-Coriolis equations, which obtained by replacing the Laplacian operator in the Navier-Stokes-Coriolis equation by the more general operator $(-\Delta)^{\alpha}$ with $\alpha>0$. We introduce function spaces of the Besove type characterized by the time evolution semigroup associated with the general linear Stokes-Coriolis operator. Next, we establish the unique existence of global in time mild solutions for small initial data belonging to our function spaces characterized by semigroups in both the scaling subcritical and critical settings.
\end{abstract}

Keywords: Cauchy problem; The generalized Navier-Stokes-Coriolis equation; Global wellposedness.

\section{Introduction}

In this paper, we study the initial value problem for the fractional Navier-StokesCoriolis equation in $\mathbb{R}^{3}$, describing the rotating flow of an incompressible viscous fluid:

$$
\begin{cases}\partial_{t} u+v(-\Delta)^{\alpha} u+(u \cdot \nabla) u+\Omega e_{3} \times u+\nabla p=0, & \text { in } \mathbb{R}^{3} \times(0, \infty), \\ \operatorname{div} u=0, & \text { in } \mathbb{R}^{3} \times(0, \infty), \\ u(0, x)=u^{0}(x), & \text { in } \mathbb{R}^{3},\end{cases}
$$

where $\alpha>0$ is the 'strength of dissipation', $u=u(t, x)=\left(u_{1}(t, x), u_{2}(t, x), u_{3}(t, x)\right)$ and $p=p(t, x)$ denote the unkown velocity field and the unknow pressure, respectively, while $u_{0}=u_{0}(x)=\left(u_{0}^{1}(x), u_{0}^{2}(x), u_{0}^{3}(x)\right)$ denote the initial velocity field. Here, $\Omega \in \mathbb{R}$ is the Coriolis parameter, which represents the speed of rotation around the vertical unit vector $e_{3}=(0,0,1)$. Moreover, $\partial t$ and $\Delta=\sum_{j=1}^{3} \partial_{x_{j}}^{2}$ are the partial derivative with respect to $t$ and the Laplacian with respect to $x=\left(x_{1}, x_{2}, x_{3}\right)$, respectively. We define

$$
\mathcal{F}\left((-\Delta)^{\alpha} u\right)(t, \xi)=|\xi|^{2 \alpha} \mathcal{F}(u)(t, \xi),
$$

where $\mathcal{F} u$ is the Fourier transform of $u$ with respect to spatial variable $x$. More details on $(-\Delta)^{\alpha}$ can be found in [23].

In the case $\Omega=0$, the system (1) corresponds to the generalized incompressible Navier-Stokes equation(GNS). Lions [21] proved the global existence of the classical solutions to the GNS equations when $\alpha \geq \frac{5}{4}$ in dimensional 3. Wu [24] obtained similar result for $\alpha \geq \frac{1}{2}+\frac{n}{4}$ in dimensional $n$. Wu also proved the existence of global-in-time weak solutions in [24]. For the existence of strong solutions, we note that the GNS equations have the following scaling invariant property.

$$
u_{\lambda}(t, x)=\lambda^{2 \alpha-1} u\left(\lambda^{2 \alpha} t, \lambda x\right), \quad p_{\lambda}(t, x)=\lambda^{4 \alpha-2} p\left(\lambda^{2 \alpha} t, \lambda x\right),
$$




$$
u_{0, \lambda}(x)=\lambda^{2 \alpha-1} u_{0}(\lambda x)
$$

Using the above scaling invariant property, $\mathrm{Wu}[25,26]$ consider the existence of solution to GNS equations in $\dot{B}_{p, q}^{1+\frac{n}{p}-2 \alpha}\left(\mathbb{R}^{3}\right)$. Zhai [27] proved the well-posedness of GNS equations in the critical space close to $\dot{B}_{\infty, \infty}^{-(2 \alpha-1)}\left(\mathbb{R}^{n}\right)$ with $\alpha \in\left(\frac{1}{2}, 1\right)$. Sun and Ding [7] studied dispersive effect of the Coriolis force and local well-posedness for the fractional NavierStokes-Coriolis system (1). In addition, Ding and Sun [8] also established the global existence and uniqueness of regular solutions in spatial variable for the higher-order elliptic Navier-Stokes system.

When $\alpha=1$, Eq.(1) become the 3D incompressible Navier-Stokes equation with Coriolis force

$$
\begin{cases}\partial_{t} u-\Delta u+\Omega e_{3} \times u+(u \cdot \nabla) u+\nabla p=0, & \text { in } \mathbb{R}^{3} \times(0, \infty), \\ \operatorname{div} u=0, & \text { in } \mathbb{R}^{3} \times(0, \infty), \\ u(0, x)=u^{0}(x), & \text { in } \mathbb{R}^{3}\end{cases}
$$

For the global existence of solutiens to (2), Chemin, Desjardins, Gallagher and Grenier [5,6] proved that for every initial velocity $u_{0} \in L^{2}\left(\mathbb{R}^{2}\right)^{3}+\dot{H}^{\frac{1}{2}}\left(R^{3}\right)^{3}$ there exists a positive parameter $\Omega_{0}=\Omega_{0}\left(u_{0}\right)$ such that for every $\Omega \in \mathbb{R}$ with $|\Omega| \geq \Omega_{0}$, (2) posseses a unique global solution. Iwabuchi and Takada [12,14] and Koh, Lee and Takada [18] proved the global and long-time existence of the unique solution in the homogeneous sobolev spaces $\dot{H}^{s}\left(\mathbb{R}^{3}\right)$ for $s>\frac{1}{2}$ if the speed of rotation is sufficiently large. Ohyama [15] proved global well-posedness for the Navier-Stokes equations with the Coriolis force in function spaces characterized by semigroups. Babin, Mahalov and Nicolaenko [1-3] obtained the global existence and regularity of the solution to (2) for large $|\Omega|$ with the spatial periodic initial velocity field. Giga, Inui, Mahalov and Saal [10] proved the uniform global well-posedness to (2) for the small initial data in the scaling invariant space $F M_{0}^{-1}\left(\mathbb{R}^{3}\right)$. Hieber and Shibata [11] proved the small initial data uniform global well-posedness to (2) in the scaling critical Sobolev space $\dot{H}^{\frac{1}{2}}\left(\mathbb{R}^{3}\right)$. We refer to $[13,19]$ for the uniform well-posedness results in other scaling invariant spaces.

In this paper, we mainly consider the case $\alpha>0$, i.e., prove the global wellposedness of (1) in some function spaces characterized by the time evolution semigroup generated by the linear operator $(-\Delta)^{\alpha}+\Omega \mathbb{P} e_{3} \times \mathbb{P}$. Where $\mathbb{P}$ is the Helmholtz projection.

To study the problem (1), we consider the following equivalent integral equation

$$
u(t)=T_{\Omega}(t) u_{0}-\int_{0}^{t} T_{\Omega}(t-\tau) \mathbb{P} \nabla \cdot(u(\tau) \otimes u(\tau)) d \tau,
$$

where $\mathbb{P}=\left(\delta_{i j}+R_{i} R_{j}\right)_{1 \leq i, j \leq 3}$ denotes the Helmholtz projection onto the divergence free vector fields, $T_{\Omega}(t)=e^{t\left(-(-\Delta)^{\alpha}-\Omega \mathbb{P} e_{3} \times \mathbb{P}\right)}$ denotes the semigroup associated with linearized problem of (1), which is given explicitly by

$$
T_{\Omega}(t) f:=\mathcal{F}^{-1}\left[\cos \left(\Omega \frac{\xi 3}{|\xi|} t\right) e^{-t|\xi|^{2 \alpha}} I \hat{f}(\xi)+\sin \left(\Omega \frac{\xi 3}{|\xi|} t\right) e^{-t|\xi|^{2 \alpha}} R(\xi) \hat{f}(\xi)\right]
$$

for $t \geq 0$ and divergence-free vector field $f$. Here, $I$ is the identity matrix in $\mathbb{R}^{3}, R(\xi)$ is the skew-symmetric matrix defined by

$$
R(\xi):=\frac{1}{|\xi|}\left(\begin{array}{ccc}
0 & \xi_{3} & -\xi_{2} \\
-\xi_{3} & 0 & \xi_{1} \\
\xi_{2} & -\xi_{1} & 0
\end{array}\right) \quad \text { for } \xi \in \mathbb{R}^{3} \backslash\{0\} .
$$


We refer to $[1,3,11]$ for the derivation of the explicit form of $T_{\Omega}(t) f$. We say that $u$ is a mild solution to problem (1) if $u$ satisfies the integral equation (3) in an appropriate function space.

Now, let us introduce our function space $X_{\Omega}^{s, p, \theta}\left(\mathbb{R}^{3}\right)$ and $X_{\Omega}^{p}\left(\mathbb{R}^{3}\right)$ of the Besov type characterized by the linear semigroup $T_{\Omega}(t)=e^{t\left(-(-\Delta)^{\alpha}-\Omega \mathbb{P} e_{3} \times \mathbb{P}\right)}$ in (4). We denote the set of all tempered distributions by $\mathscr{S}^{\prime}\left(\mathbb{R}^{3}\right)$.

Definition 1. Let $\Omega \in \mathbb{R}, \alpha>0$.

(i) For $s \in \mathbb{R}$ and $1 \leq p, \theta \leq \infty$, the function space $X_{\Omega}^{s, p, \theta}\left(\mathbb{R}^{3}\right)$ is defined as follows:

$$
\begin{aligned}
X_{\Omega}^{s, p, \theta}\left(\mathbb{R}^{3}\right) & =\left\{f \in \mathscr{S}^{\prime} \mid\|f\|_{X_{\Omega}^{s, p, \theta}}<\infty\right\}, \\
\|f\|_{X_{\Omega}^{s, p, \theta}} & =\left\|T_{\Omega}(t) f\right\|_{L_{t}^{\theta}\left(0, \infty ; \dot{W}_{x}^{s, p}\left(\mathbb{R}^{3}\right)\right)} .
\end{aligned}
$$

(ii) For $1 \leq p \leq \infty$, the function space $X_{\Omega}^{p}\left(\mathbb{R}^{3}\right)$ is defined as follows:

$$
\begin{aligned}
& X_{\Omega}^{p}\left(\mathbb{R}^{3}\right)=\left\{f \in \mathscr{S}^{\prime} \mid\|f\|_{X_{\Omega}^{p}}<\infty\right\}, \\
& \|f\|_{X_{\Omega}^{p}}=\sup _{t>0} t^{\frac{1}{2 \alpha}\left(1-\frac{3}{p}\right)}\left\|T_{\Omega}(t) f\right\|_{L^{p}} .
\end{aligned}
$$

Remark 1. (i) If $\Omega=0, T_{0}(t) f=e^{-t(-\Delta)^{\alpha}}$. Then, for $1 \leq \theta<\infty$, according to [22], there is

$$
\|f\|_{X_{0}^{s, p, \theta}}=\left\|t^{\frac{1}{\theta}}\right\|(-\Delta)^{\frac{s}{2}} e^{-t(-\Delta)^{\alpha}} f\left\|_{L^{p}}\right\|_{L^{\theta}\left(0, \infty ; \frac{d t}{t}\right)} \simeq\left\|(-\Delta)^{\frac{s}{2}} f\right\|_{\dot{B}_{p, \theta}^{-\frac{2 \alpha}{\theta}}}=\|f\|_{\dot{B}_{p, \theta}^{s-\frac{2 \alpha}{\theta}}} .
$$

We also see that for $3<p \leq \infty$

$$
\|f\|_{X_{0}^{p}}=\left\|t^{\frac{1}{2 \alpha}\left(1-\frac{3}{p}\right)}\right\| e^{-t(-\Delta)^{\alpha}} f\left\|_{L^{p}}\right\|_{L^{\infty}\left(0, \infty ; \frac{d t}{t}\right)} \simeq\|f\|_{\dot{B}_{p, \infty}^{-1+\frac{3}{p}}} .
$$

Therefore, the function space $X_{\Omega}^{s, p, \theta}\left(\mathbb{R}^{3}\right)$ and $X_{\Omega}^{p}\left(\mathbb{R}^{3}\right)$ can be regard as one of the generalizations of the Besove space $\dot{B}_{p, \theta}^{s-\frac{2 \alpha}{\theta}}\left(\mathbb{R}^{3}\right)$ and $\dot{B}_{p, \infty}^{-1+\frac{3}{p}}\left(\mathbb{R}^{3}\right)$, respectively.

(ii) For $3 \leq p<\infty$, we set $\frac{1}{q}:=\frac{1}{p}+\frac{1}{6}$. We see that $2 \leq q<6$ and the Sobolev embedding $\dot{W}^{\frac{1}{2}}, q\left(\mathbb{R}^{3}\right) \hookrightarrow L^{p}\left(\mathbb{R}^{3}\right)$ holds. Therefore, it follows from the Plancherel theorem and Lemma 6 that

$$
\begin{aligned}
\left\|T_{\Omega}(t) f\right\|_{L^{p}} & \leq\left\|T_{\Omega}(t) f\right\|_{\dot{W}^{\frac{1}{2}, q}}=\left\|(-\Delta)^{\frac{1}{4}} T_{\Omega}(t) f\right\|_{L^{q}} \\
& \leq t^{-\frac{3}{2 \alpha}\left(\frac{1}{2}-\frac{1}{q}\right)}\left\|(-\Delta)^{\frac{1}{4}} f\right\|_{L^{2}} \\
& =C t^{-\frac{1}{2 \alpha}\left(1-\frac{3}{p}\right)}\|f\|_{\dot{H}^{\frac{1}{2}}}
\end{aligned}
$$

for all $t>0$ and $\Omega \in \mathbb{R}$. Hence the continuous embedding $\dot{H}^{\frac{1}{2}}\left(\mathbb{R}^{3}\right) \hookrightarrow X_{\Omega}^{p}\left(\mathbb{R}^{3}\right)$ holds for all $\Omega \in \mathbb{R}$ and $3 \leq p<\infty$.

In this paper, we show the unique existence of global in time solutions in the subcritical spaces $X_{\Omega}^{s, p, \theta}\left(\mathbb{R}^{3}\right)$ with $\frac{2 \alpha}{\theta}+\frac{3}{p}<s+(2 \alpha-1), \frac{1}{2}<\alpha \leq 1$, and the uniform global well-posedness in the scaling critical spaces $X_{\Omega}^{p}\left(\mathbb{R}^{3}\right)$ with $\frac{3}{2 \alpha-1}<p \leq 4, \frac{7}{8}<\alpha \leq$ $\frac{5}{4}$.

The following is our result in scaling subcritical cases. 
Theorem 1. Let $\alpha, s, p$, and $\theta$ satisfy

$$
\begin{aligned}
\frac{1}{2}<\alpha & \leq 1, \quad 3-3 \alpha<s<\frac{3(15-4 \alpha)}{2(9+8 \alpha)} \\
\frac{1}{3}+\frac{s}{9} & \leq \frac{1}{p}<\min \left\{\frac{1}{4}+\frac{5}{16 \alpha}-\frac{s}{8 \alpha}, \frac{2 \alpha-1}{3}+\frac{s}{3}\right\} \\
\max \left\{0, \frac{2}{p}-\frac{1}{2 \alpha}\left(1+\frac{3}{p}-s\right)\right\} & <\frac{1}{\theta}<\min \left\{\frac{1}{2}, 1-\frac{1}{2 \alpha}\left(1+\frac{3}{p}-s\right), \frac{1}{2}+\frac{1}{8 \alpha}-\frac{3}{2 \alpha p}+\frac{s}{4 \alpha}\right\} .
\end{aligned}
$$

Then, there exists positive constants $C=C(\alpha, s, p, \theta)$ such that for $\Omega \in \mathbb{R} \backslash\{0\}$ and for initial velocity $u_{0} \in X_{\Omega}^{s, p, \theta}\left(\mathbb{R}^{3}\right)^{3} \cap \dot{H}^{s}\left(\mathbb{R}^{3}\right)^{3}$ satisfying $\operatorname{div} u_{0}=0$ and

$$
\left\|u_{0}\right\|_{X_{\Omega}^{s, p, \theta}} \leq C|\Omega|^{1-\frac{1}{2 \alpha}\left(1+\frac{3}{p}-s\right)-\frac{1}{\theta}}
$$

(2) possesses a unique mild solution

$$
u \in L^{\theta}\left(0, \infty ; \dot{W}^{s, p}\left(\mathbb{R}^{3}\right)^{3}\right) \cap C\left([0, \infty) ; \dot{H}^{s}\left(\mathbb{R}^{3}\right)^{3}\right)
$$

satisfying $\operatorname{div} u=0$.

Remark 2. In the case $\Omega=0$, as we have seen in (5), there hold

$$
\left\|u_{0}\right\|_{X_{0}^{s, p, \theta}} \simeq\left\|u_{0}\right\|_{\dot{B}_{p, \theta}^{s-\frac{2 \alpha}{\theta}}},\left\|\lambda^{2 \alpha-1} u_{0}(\lambda \cdot)\right\|_{\dot{B}_{p, \theta}^{s-\frac{2 \alpha}{\theta}}}=\lambda^{2 \alpha-1+s-\left(\frac{2 \alpha}{\theta}+\frac{3}{p}\right)}\left\|u_{0}\right\|_{\dot{B}_{p, \theta}^{s-\frac{2 \alpha}{\theta}}} .
$$

for dyadic number $\lambda>0$. Since $\frac{2 \alpha}{\theta}+\frac{3}{p}<s+(2 \alpha-1)$ by our assumption (9), the function spaces $X_{\Omega}^{s, p, \theta}\left(\mathbb{R}^{3}\right)$ in Theorem 1 correspond to the subcritical cases from the viewpoint of the scaling properties. In the case $\alpha=1$, Ohyama [15] proved unique existence of global in time mild solutions for every $\Omega \in \mathbb{R} \backslash\{0\}$ and $u_{0} \in X_{\Omega}^{s, p, \theta}\left(\mathbb{R}^{3}\right)^{3} \cap \dot{H}^{s}\left(\mathbb{R}^{3}\right)^{3}$. Hence, we generalize their results for $\alpha=1$.

Our main result in the scaling critical case reads as follows:

Theorem 2. Let $\alpha, s, p$ satisfy

$$
\frac{7}{8}<\alpha \leq \frac{5}{4}, \quad 0 \leq s<2 \alpha-1, \quad \max \left\{\frac{1}{4}, \frac{s}{3}\right\} \leq \frac{1}{p}<\frac{2 \alpha-1}{3}
$$

Then, there exists a positive constant $\delta=\delta(\alpha, s, p$,$) independent of \Omega \in \mathbb{R}$ such that for the initial velocity $u_{0} \in X_{\Omega}^{p}\left(\mathbb{R}^{3}\right)^{3} \cap \dot{H}^{s}\left(\mathbb{R}^{3}\right)^{3}$ satisfying $\operatorname{div} u_{0}=0$ and

$$
\left\|u_{0}\right\|_{X_{\Omega}^{p}} \leq \delta
$$

(1) possesses a unique mild solution

$$
u \in B C\left([0, \infty) ; \dot{H}^{s}\left(\mathbb{R}^{3}\right)^{3}\right) \text { satisfying } \sup _{t>0} t^{\frac{1}{2 \alpha}\left(1-\frac{3}{p}\right)}\|u(t)\|_{L^{p}} \leq 2\left\|u_{0}\right\|_{X_{\Omega}^{p}}
$$

and $\operatorname{div} u=0$ for all $\Omega \in \mathbb{R}$.

Remark 3. (i) In [15], Ohyama proved the uniform global well-posedness in $X_{\Omega}^{p}\left(\mathbb{R}^{3}\right)$ with $\frac{3}{2 \alpha-1}<p \leq 4$. Here, we generalize their results for $\alpha=1$. 
The rest of this paper is organized as follows. In Section 2, we collect some basic facts on Littlewood-Paley theory, and show some new linear estimates for semigroup $\left\{T_{\Omega}(t)\right\}_{t \geq 0}$. In Section 3, we establish the bilinear estimates for the Duhamel terms in (4). Finally, we present the proofs of the main results.

\section{Linear Estimates}

Let $\mathscr{S}\left(\mathbb{R}^{3}\right)$ be the Schwartz space. First, we recall the homogeneous LittlewoodPaley decomposition. Let $\varphi_{0} \in \mathscr{S}\left(\mathbb{R}^{3}\right)$ satisfy the following properties:

$$
\begin{gathered}
0 \leq \hat{\varphi}_{0}(\xi) \leq 1 \quad \text { for all } \xi \in \mathbb{R}^{3}, \operatorname{supp} \hat{\varphi}_{0} \subset:=\left\{\xi \in \mathbb{R}^{3}: \frac{1}{2} \leq|\xi| \leq 2\right\}, \\
\text { and } \sum_{j \in \mathbb{Z}} \hat{\varphi}_{0}\left(2^{-j} \xi\right)=1 \quad \text { for all } \xi \in \mathbb{R}^{3} \backslash\{0\} .
\end{gathered}
$$

where $\varphi_{j}(x):=2^{3 j} \varphi_{0}\left(2^{j} x\right)$. Then, we define the Littlewood-Paley operators $\left\{\Delta_{j}\right\}_{j \in z}$ by $\Delta_{j} f:=\varphi_{j} * f$ for $f \in \mathscr{S}^{\prime}\left(\mathbb{R}^{3}\right)$.

Now, we introduce the definitions of homogeneous Besov space $\dot{B}_{p, q}^{s}\left(\mathbb{R}^{3}\right)$.

Definition 2. Let $s \in \mathbb{R}$ and $1 \leq p, q \leq \infty$. Then, we define the homogeneous Besov space $\dot{B}_{p, q}^{s}\left(\mathbb{R}^{3}\right) b y$

$$
\begin{gathered}
\dot{B}_{p, q}^{s}\left(\mathbb{R}^{3}\right):=\left\{f \in \mathscr{S}^{\prime}\left(\mathbb{R}^{3}\right) \mid\|f\|_{\dot{B}_{p, q}^{s}}<+\infty\right\}, \\
\|f\|_{\dot{B}_{p, q}^{s}}:=\left(\sum_{j \in \mathbb{Z}} 2^{j s q}\left\|\Delta_{j} u\right\|_{L^{p}}^{q}\right)^{\frac{1}{q}} .
\end{gathered}
$$

Next, we define the operators $\mathcal{G}_{ \pm}(t)$ by

$$
\mathcal{G}_{ \pm}(t) f(x):=e^{ \pm i t \frac{D_{3}}{|D|}} f(x):=\int_{\mathbb{R}^{3}} e^{i x \cdot \xi \pm i t \frac{\xi_{3}}{|\xi|}} \hat{f}(\xi) d \xi \quad \text { for } x \in \mathbb{R}^{3} \text { and } t \in \mathbb{R}
$$

Then, we can rewrite the operator $T_{\Omega}(t)$ as

$$
T_{\Omega}(t) f=\frac{1}{2} \mathcal{G}_{+}(\Omega t)\left[e^{-t(-\Delta)^{\alpha}}(I+\mathcal{R}) f\right]+\frac{1}{2} \mathcal{G}_{-}(\Omega t)\left[e^{-t(-\Delta)^{\alpha}}(I-\mathcal{R}) f\right] .
$$

for all $t \geq 0$, where $\mathcal{R}$ denotes the matrix of singular integral operators defined by

$$
\mathcal{R}:=\left(\begin{array}{ccc}
0 & R_{3} & -R_{2} \\
-R_{3} & 0 & R_{1} \\
R_{2} & -R_{1} & 0
\end{array}\right)
$$

First, we recall the behavior of the fractional order heat semigroup $e^{-t(-\Delta)^{\alpha}}$ in Lebesgue spaces.

Lemma 1. (Miao-Yuan-zhang[22]) Let $1 \leq r \leq p \leq \infty$ and $f \in L^{r}\left(\mathbb{R}^{n}\right)$. Then, $e^{-t(-\Delta)^{\alpha}}$ satisfies the estimates

$$
\begin{gathered}
\left\|e^{-t(-\Delta)^{\alpha}} f(x)\right\|_{L^{p}} \leq C t^{-\frac{n}{2 \alpha}\left(\frac{1}{r}-\frac{1}{p}\right)}\|f\|_{L^{r}} \\
\left\|(-\Delta)^{\frac{\beta}{2}} e^{-t(-\Delta)^{\alpha}} f(x)\right\|_{L^{p}} \leq C t^{-\frac{\beta}{2 \alpha}-\frac{n}{2 \alpha}\left(\frac{1}{r}-\frac{1}{p}\right)}\|f\|_{L^{r}}
\end{gathered}
$$

for $\alpha>0$ and $\beta>0$.

Second, we recall the linear estimates for the semigroup $e^{-t(-\Delta)^{\alpha}}$ in homogeneous Bessov spaces as follows: 
Lemma 2. (Sun-Ding[7]) Let $-\infty<s_{0} \leq s_{1}<\infty, 1 \leq p, q \leq \infty$. Then, there exists a positive constant $C=C\left(s_{0}, s_{1}\right)$ such that

$$
\left\|e^{-t(-\Delta)^{\alpha}} f\right\|_{\dot{B}_{p, q}^{s_{1}}} \leq C t^{-\frac{1}{2 \alpha}\left(s_{1}-s_{0}\right)}\|f\|_{\dot{B}_{p, q}^{s_{0}}}
$$

for all $t>0, \alpha>0,1 \leq p \leq \infty$ and $f \in \dot{B}_{p, q}^{s_{0}}\left(\mathbb{R}^{3}\right)$.

Lemma 3. (Kozono et al.[20]) Let $-\infty<s_{0} \leq s_{1}<\infty, 1 \leq p_{0} \leq p_{1} \leq \infty, 1 \leq q \leq \infty$. Then, there exists a positive constant $C=C\left(s_{0}, s_{1}, p_{0}, p_{1}\right)$ such that

$$
\left\|e^{-t(-\Delta)^{\alpha}} f\right\|_{\dot{B}_{p_{1}, q}^{s_{1}}} \leq C t^{-\frac{1}{2 \alpha}\left(s_{1}-s_{0}\right)-\frac{3}{2 \alpha}\left(\frac{1}{p_{0}}-\frac{1}{p_{1}}\right)}\|f\|_{\dot{B}_{p_{0}, q}^{s_{0}}}
$$

for all $t>0, \alpha>0, f \in \dot{B}_{p, q}^{s_{0}}\left(\mathbb{R}^{3}\right)$.

The following is our key dispersive estimates for the operator $\mathcal{G}_{ \pm}(\tau)$.

Lemma 4. (Koh, Lee and Takada [18]) For $2 \leq p \leq \infty$, there exists a positive constant $C=C(p)$ such that

$$
\left\|\mathcal{G}_{ \pm}(\tau) f\right\|_{\dot{B}_{p, q}^{s}} \leq C(1+|\tau|)^{-\left(1-\frac{2}{p}\right)}\|f\|_{\dot{B}_{p^{\prime}, q}^{s+3\left(1-\frac{2}{p}\right)}}
$$

for all $\tau \in \mathbb{R}, s \in \mathbb{R}, 1 \leq q \leq \infty$, and $f \in \dot{B}_{p^{\prime}, q}^{s+3\left(1-\frac{2}{p}\right)}\left(\mathbb{R}^{3}\right)$ where $\frac{1}{p}+\frac{1}{p^{\prime}}=1$.

By combing this with the Plancherel theorem, we obtain the linear estimates for the semigroup $T_{\Omega}(t)$.

Lemma 5. Let $-\infty<s_{0} \leq s_{1}<\infty, 1 \leq p_{0} \leq 2 \leq p_{1} \leq \infty$ and $1 \leq q \leq \infty$. Then, there exists a positive constant $\bar{C}=C\left(\alpha, s_{0}, s_{1}, p_{0}, p_{1}\right)$ such that

$$
\left\|T_{\Omega}(t) f\right\|_{\dot{B}_{p_{1}, q}^{s_{1}}} \leq C t^{-\frac{1}{2 \alpha}\left(s_{1}-s_{0}\right)-\frac{3}{2 \alpha}\left(\frac{1}{p_{0}}-\frac{1}{p_{1}}\right)}\|f\|_{\dot{B}_{p_{0}, q}^{s_{0}}}
$$

for $t>0, \alpha>0$, and $f \in \dot{B}_{p_{0}, q}^{s_{0}}\left(\mathbb{R}^{3}\right)$.

Proof. Since $\mathcal{R}$ is bounded in $L^{2}\left(\mathbb{R}^{3}\right)$, it follows from the Plancherel theorem, $L^{p_{0}}-L^{2}$ estimate for the semigroup $e^{-t(-\Delta)^{\alpha}}$, Lemma 3 and Lemma 4 that

$$
\begin{aligned}
& \left\|e^{-\frac{t}{2}(-\Delta)^{\alpha}} \mathcal{G}_{ \pm}(\Omega t)\left[e^{-\frac{t}{2}(-\Delta)^{\alpha}}(I \pm \mathcal{R}) f\right]\right\|_{\dot{B}_{p_{1}, q}^{s_{1}}} \\
\leq & C t^{-\frac{1}{2 \alpha}\left(s_{1}-s_{0}\right)-\frac{3}{2 \alpha}\left(\frac{1}{2}-\frac{1}{p_{1}}\right)}\left\|\mathcal{G}_{ \pm}(\Omega t)\left[e^{-\frac{t}{2}(-\Delta)^{\alpha}} f\right]\right\|_{\dot{B}_{2, q}^{s_{0}}} \\
\leq & C t^{-\frac{1}{2 \alpha}\left(s_{1}-s_{0}\right)-\frac{3}{2 \alpha}\left(\frac{1}{2}-\frac{1}{p_{1}}\right)}\left\|e^{-\frac{t}{2}(-\Delta)^{\alpha}} f\right\|_{\dot{B}_{2, q}^{s_{0}}} \\
\leq & C t^{-\frac{1}{2 \alpha}\left(s_{1}-s_{0}\right)-\frac{3}{2 \alpha}\left(\frac{1}{p_{0}}-\frac{1}{p_{1}}\right)}\|f\|_{\dot{B}_{p_{0}, q}^{s_{0}}} .
\end{aligned}
$$

Thus, we complete the Proof of Lemma 5.

By Lemma 4, the continuous embeddings $L^{p}\left(\mathbb{R}^{3}\right) \hookrightarrow \dot{B}_{p, 2}^{0}\left(\mathbb{R}^{3}\right)(1<p \leq 2)$, $\dot{B}_{p, 2}^{0}\left(\mathbb{R}^{3}\right) \hookrightarrow L^{p}\left(\mathbb{R}^{3}\right)(2 \leq p<\infty)$, and the fact $\dot{H}^{s}\left(\mathbb{R}^{3}\right)=\dot{B}_{2,2}^{s}$ (see, for examples, [4] ), we obtain the following Lemma 6 . 
Lemma 6. Let $0 \leq s<\infty, 1<p_{0} \leq 2 \leq p_{1}<\infty$ and $\beta=\left(\beta_{1}, \beta_{2}, \beta_{3}\right) \in(\mathbb{N} \cup\{0\})^{3}$, Then, there exists a positive constant $C=C\left(\alpha, s, p_{0}, p_{1}, \beta\right)$ such that

$$
\begin{gathered}
\left\|\partial_{x}^{\beta} T_{\Omega}(t) f\right\|_{\dot{H}^{s}} \leq C t^{-\frac{1}{2 \alpha}(s+|\beta|)-\frac{3}{2 \alpha}\left(\frac{1}{p_{0}}-\frac{1}{2}\right)}\|f\|_{L^{p_{0}}}, \\
\left\|\partial_{x}^{\beta} T_{\Omega}(t) f\right\|_{L^{p_{1}}} \leq C t^{-\frac{\beta}{2 \alpha}-\frac{3}{2 \alpha}\left(\frac{1}{p_{0}}-\frac{1}{p_{1}}\right)}\|f\|_{L^{p_{0}}}
\end{gathered}
$$

for $t>0, \alpha>0$ and $f \in L^{p_{0}}\left(\mathbb{R}^{3}\right)$.

We next recall the uniform boundedness of $T_{\Omega}(t)$ in $\dot{H}^{s}\left(\mathbb{R}^{3}\right)$ with respect to $t>0$ and $\Omega \in \mathbb{R}$.

Lemma 7. (Sun-Ding[7]) For $s \in \mathbb{R}$, there exists a positive constant $C=C(s)$ such that

$$
\left\|T_{\Omega}(t) f\right\|_{\dot{H}^{s}} \leq C\|f\|_{\dot{H}^{s}}
$$

for all $t>0, \Omega \in \mathbb{R}$ and $f \in \dot{H}^{s}\left(\mathbb{R}^{3}\right)^{3}$.

Lemma 8. Let $\alpha, p, q$ and $\theta$ satisfy $\frac{1}{2}<\alpha<\frac{5}{4}, 2<p<\frac{3}{2-\alpha}$ and $1-\frac{1}{p} \leq \frac{1}{q}<\frac{2 \alpha-1}{3}+\frac{1}{p}$,

$$
\max \left\{0,1-\frac{1}{2 \alpha}-\frac{3}{2 \alpha}\left(\frac{1}{q}-\frac{1}{p}\right)-\left(1-\frac{2}{p}\right)\right\}<\frac{1}{\theta} \leq \min \left\{\frac{1}{2}, 1-\frac{1}{2 \alpha}-\frac{3}{2 \alpha}\left(\frac{1}{q}-\frac{1}{p}\right)\right\} .
$$

Then, there exists a positive constant $C=C(\alpha, p, q, \theta)$ such that

$$
\left\|\int_{0}^{t} T_{\Omega}(t-\tau) \mathbb{P} \nabla f(\tau) d \tau\right\|_{L^{\theta}\left(0, \infty ; L^{p}\right)} \leq C|\Omega|^{-\left\{1-\frac{1}{2 \alpha}-\frac{3}{2 \alpha}\left(\frac{1}{q}-\frac{1}{p}\right)-\frac{1}{\theta}\right\}}\|f\|_{L^{\frac{\theta}{2}}\left(0, \infty ; L^{q}\right)}
$$

for all $\Omega \in \mathbb{R} \backslash\{0\}$ and $f \in L^{\frac{\theta}{2}}\left(0, \infty ; L^{q}\right)\left(\mathbb{R}^{3}\right)$. In particular, in the case $\frac{1}{\theta}=1-\frac{1}{2 \alpha}-$ $\frac{3}{2 \alpha}\left(\frac{1}{q}-\frac{1}{p}\right),(2.2)$ holds for all $\Omega \in \mathbb{R}$.

Proof. Since $\mathbb{P}$ is bounded in $L^{q}\left(\mathbb{R}^{3}\right)$, it follows from $\dot{B}_{p, 2}^{0}\left(\mathbb{R}^{3}\right) \hookrightarrow L^{p}\left(\mathbb{R}^{3}\right)(2 \leq p<\infty)$, Lemma 4, Lemma 3 and Lemma 1 that

$$
\left\|\int_{0}^{t} T_{\Omega}(t-\tau) \mathbb{P} \nabla f(\tau) d \tau\right\|_{L^{\theta}\left(0, \infty ; L^{p}\right)} \leq C\left\|\int_{0}^{t} k_{\Omega}(t-\tau)\right\| f(\tau)\left\|_{L^{q}} d \tau\right\|_{L_{t}^{\theta}(0, \infty)^{\prime}}
$$

where

$$
k_{\Omega}(t):=\{1+|\Omega| t\}^{-\left(1-\frac{2}{p}\right)} t^{-\frac{3}{2 \alpha}\left(\frac{1}{q}-\frac{1}{p}\right)-\frac{1}{2 \alpha}}
$$

If $\frac{1}{\theta}<1-\frac{1}{2 \alpha}-\frac{3}{2 \alpha}\left(\frac{1}{q}-\frac{1}{p}\right)$, we have $\left\|k_{\Omega}\right\|_{L^{\theta^{\prime}}} \leq C|\Omega|^{-\left\{1-\frac{1}{2 \alpha}-\frac{3}{2 \alpha}\left(\frac{1}{q}-\frac{1}{p}\right)-\frac{1}{\theta}\right\}}$. According Young inequality, $\frac{1}{\theta}=\frac{1}{\theta^{\prime}}+\frac{2}{\theta}-1$, we have

$$
\left\|\int_{0}^{t} T_{\Omega}(t-\tau) \mathbb{P} \nabla f(\tau) d \tau\right\|_{L^{\theta}\left(0, \infty ; L^{p}\right)} \leq C|\Omega|^{-\left\{1-\frac{1}{2 \alpha}-\frac{3}{2 \alpha}\left(\frac{1}{q}-\frac{1}{p}\right)-\frac{1}{\theta}\right\}}\|f\|_{L^{\frac{\theta}{2}}\left(0, \infty ; L^{q}\right)} .
$$

If $\frac{1}{\theta}=1-\frac{1}{2 \alpha}-\frac{3}{2 \alpha}\left(\frac{1}{q}-\frac{1}{p}\right)$. So, by the Hardy-Littlewood-Sobolev inequality, we see

$$
\left\|\int_{0}^{t} T_{\Omega}(t-\tau) \mathbb{P} \nabla f(\tau) d \tau\right\|_{L^{\theta}\left(0, \infty ; L^{p}\right)} \leq C\|f\|_{L^{\frac{\theta}{2}}\left(0, \infty ; L^{q}\right)}
$$

for all $\Omega \in \mathbb{R}$. This completes the proof of Lemma 2.9 . 


\section{Bilinear Estimates}

In this section, we obtain bilinear estimates which are used to handle the Duhamel terms in (3). Firstly, we recall the following bilinear estimates in the homogeneous Sobolev spaces.

Lemma 9. (Koh, Lee and Takada [18]) Let $s, p$, and $q$ satisfy $0 \leq s<3, \frac{s}{3}<\frac{1}{p}<\frac{1}{2}+\frac{s}{6}$ and $\frac{1}{q}=\frac{2}{p}-\frac{s}{3}$. Then, there exists a positive constant $C=C(s, p)$ such that

$$
\|f g\|_{\dot{W}^{s, q}} \leq C\|f\|_{\dot{W}^{s, p}}\|g\|_{\dot{W}^{s, p}}
$$

for $f, g \in \dot{W}^{s, p}\left(\mathbb{R}^{3}\right)$.

Now, we consider the bilinear estimates for the Duhamel term in (3). Let us set

$$
N(u, v)(t):=\int_{0}^{t} T_{\Omega}(t-\tau) \mathbb{P} \nabla \cdot(u(\tau) \otimes u(\tau)) d \tau, t \geq 0 .
$$

We define $\|u\|_{Z_{1}}=\|u\|_{L^{\theta}\left(0, \infty ; \dot{W}^{s, p}\right)}$. Then, we obtain the bilinear estimates for $N(u, v)$ in $Z_{1}$.

Lemma 10. Let $\alpha, s, p, \theta$ satisfy $\frac{1}{2}<\alpha \leq 1,3-3 \alpha<s<\frac{3}{2}, \frac{1}{3}+\frac{s}{9} \leq \frac{1}{p}<\min \left\{\frac{1}{2}, \frac{2 \alpha-1}{3}+\right.$ $\left.\frac{s}{3}\right\}, \max \left\{0, \frac{2}{p}-\frac{1}{2 \alpha}\left(1+\frac{3}{p}-s\right)\right\}<\frac{1}{\theta} \leq\left\{\frac{1}{2}, 1-\frac{1}{2 \alpha}\left(1+\frac{3}{p}-s\right)\right\}$. Then, there exists a positive constant $C=C(\alpha, s, p, \theta)$ such that

$$
\|N(u, v)\|_{Z_{1}} \leq C|\Omega|^{-\left\{1-\frac{1}{2 \alpha}\left(1+\frac{3}{p}-s\right)-\frac{1}{\theta}\right\}}\|u\|_{Z_{1}}\|v\|_{Z_{1}}
$$

for all $\Omega \in \mathbb{R} \backslash\{0\}$ and $u, v \in Z_{1}$. In particular, in the case $\frac{1}{\theta}=1-\frac{1}{2 \alpha}\left(1+\frac{3}{p}-s\right)$, (3.3) holds for all $\Omega \in \mathbb{R}$.

Proof. Let $\frac{1}{q}=\frac{2}{p}-\frac{s}{3}$. Hence, by Lemma 7, Lemma 8 and the Hölder inequality, we obtain

$$
\begin{aligned}
\|N(u, v)\|_{L^{\theta}\left(0, \infty ; \dot{W}^{s, p}\right)} & \leq C|\Omega|^{-\left\{1-\frac{1}{2 \alpha}\left(1+\frac{3}{p}-s\right)-\frac{1}{\theta}\right\}}\|u \otimes v\|_{L^{\frac{\theta}{2}\left(0, \infty ; \dot{W}^{s, p}\right)}} \\
& \leq C|\Omega|^{-\left\{1-\frac{1}{2 \alpha}\left(1+\frac{3}{p}-s\right)-\frac{1}{\theta}\right\}}\|u\|_{L^{\theta}\left(0, \infty ; \dot{W}^{s, p}\right)}\|v\|_{L^{\theta}\left(0, \infty ; \dot{W}^{s, p}\right)},
\end{aligned}
$$

which yields the desired estimates.

Next, we prove the bilinear estimates for Theorem 2. Let $\|\cdot\| Y_{2}$ and $\|\cdot\|_{Z_{2}}$ be defined by

$$
\|u\|_{Y_{2}}:=\sup _{t>0}\|u\|_{H^{s}}, \quad\|u\|_{Z_{2}}:=\sup _{t>0} t^{\frac{1}{2 \alpha}\left(1-\frac{3}{p}\right)}\|u(t)\|_{L^{p}} .
$$

Lemma 11. (1) Let $\frac{1}{2}<\alpha<\frac{5}{4}, 0 \leq s<2 \alpha-1$ and $\frac{s}{3} \leq \frac{1}{p}<\frac{2 \alpha-1}{3}$. Then, there exists $a$ positive constant $C_{1}=C_{1}(\alpha, s, p)$ such that

$$
\|N(u, v)\|_{Y_{2}} \leq C_{1}\|u\|_{Y_{2}}\|v\|_{Z_{2}}
$$

for all $\Omega \in \mathbb{R}$. such that

(2) Let $\frac{7}{8}<\alpha<2, \frac{3}{2 \alpha-1}<p \leq 4$. Then, there exists a positive constant $C_{2}=C_{1}(\alpha, p)$

$$
\|N(u, v)\|_{Z_{2}} \leq C_{2}\|u\|_{Z_{2}}\|v\|_{Z_{2}} .
$$

Proof. We set $r$ by

$$
\frac{1}{r}=\frac{1}{s^{*}}+\frac{1}{p}
$$


where $\frac{1}{s^{*}}=\frac{1}{2}-\frac{s}{3}$ with $2 \leq s^{*}<\frac{6}{5-4 \alpha}$ and $1<r \leq 2$. Then, it follows from Lemma 6 , the boundedness of $\mathbb{P}$ in $L^{2}\left(\mathbb{R}^{2}\right)$, and the Hölder ineqeuality that

$$
\begin{aligned}
\|N(u, v)(t)\|_{\dot{H}^{s}} & \leq \int_{0}^{t}\left\|T_{\Omega}(t-\tau) \mathbb{P} \nabla[u(\tau) \otimes v(\tau)]\right\|_{\dot{H}^{s}} d \tau \\
& \leq \int_{0}^{t} \frac{1}{(t-\tau)^{\frac{1}{2 \alpha}+\frac{3}{2 \alpha p}}}\|u(\tau) \otimes v(\tau)\|_{L^{r}} d \tau \\
& \leq \int_{0}^{t} \frac{1}{(t-\tau)^{\frac{1}{2 \alpha}+\frac{3}{2 \alpha p}}}\|u(\tau)\|_{L^{s^{*}}}\|v(\tau)\|_{L^{p}} d \tau
\end{aligned}
$$

Here, by the definitions of $\|\cdot\|_{Y_{2}}$ and $\|\cdot\|_{Z_{2}}$, the fact $\frac{1}{2 \alpha}+\frac{3}{2 \alpha p}<1$ and $\frac{1}{2 \alpha}\left(1-\frac{3}{p}\right)<1$, and the continuous embedding $\dot{H}^{s}\left(\mathbb{R}^{3}\right) \hookrightarrow L^{s^{*}}\left(\mathbb{R}^{3}\right)$, we have

$$
\begin{aligned}
\int_{0}^{t} \frac{1}{(t-\tau)^{\frac{1}{2 \alpha}+\frac{3}{2 \alpha p}}}\|u(\tau)\|_{L^{s^{*}}}\|v(\tau)\|_{L^{p}} d \tau & \leq C\|u\|_{Y_{2}}\|v\|_{Z_{2}} \int_{0}^{t} \frac{1}{(t-\tau)^{\frac{1}{2 \alpha}+\frac{3}{2 \alpha p}} \tau^{\frac{1}{2 \alpha}\left(1-\frac{3}{p}\right)} d \tau} \\
& =C\|u\|_{Y_{2}}\|v\|_{Z_{2}}
\end{aligned}
$$

where $C$ independent of $t$. Thus, we complete the proof of the inequality (18).

Next, we prove the inequality (19). Since $1<\frac{p}{2} \leq 2$, it follows from Lemma 6 , the Hölder inequality and the definitions of $\|\cdot\|_{Z_{2}}$ that

$$
\begin{aligned}
& t^{\frac{1}{2 \alpha}\left(1-\frac{3}{p}\right)}\|N(u, v)(t)\|_{L^{p}} \leq t^{\frac{1}{2 \alpha}\left(1-\frac{3}{p}\right)} \int_{0}^{t}\left\|T_{\Omega}(t-\tau) \mathbb{P} \nabla[u(\tau) \otimes v(\tau)]\right\|_{L^{p}} d \tau \\
& \leq C t^{\frac{1}{2 \alpha}\left(1-\frac{3}{p}\right)} \int_{0}^{t} \frac{1}{(t-\tau)^{\frac{1}{2 \alpha}+\frac{3}{2 \alpha p}}}\|u(\tau) \otimes v(\tau)\|_{L^{\frac{p}{2}}} d \tau \\
& \leq t^{\frac{1}{2 \alpha}\left(1-\frac{3}{p}\right)}\|u\|_{Z_{2}}\|v\|_{Z_{2}} \int_{0}^{t} \frac{1}{(t-\tau)^{\frac{1}{2 \alpha}+\frac{3}{2 \alpha p}} \tau^{\frac{1}{\alpha}\left(1-\frac{3}{p}\right)}} d \tau \\
& =C\|u\|_{Z_{2}}\|v\|_{Z_{2}}
\end{aligned}
$$

where we remark that $\frac{1}{2 \alpha}+\frac{3}{2 \alpha p}<1$ since $p>\frac{3}{2 \alpha-1}$ and $C$ independent of $t$. Thus, we complete the proof of the inequality (19).

\section{Proof of main results}

Proof of Theorem 1. It is not difficult to examine that the indices $\alpha, s, p$ and $\theta$ given in Theorem 1 satisfy the assumptions of Lemma 10. Let $\Omega \in \mathbb{R} \backslash\{0\}$. Suppose $u_{0} \in$ $X_{\Omega}^{s, p, \theta}\left(\mathbb{R}^{3}\right)^{3} \cap \dot{H}^{s}\left(\mathbb{R}^{3}\right)^{3}$ satisfies $\operatorname{div} u_{0}=0$. By the definitions of $\|\cdot\|_{Z_{1}}$, we see that $\left\|T_{\Omega}(\cdot) u_{0}\right\|_{Z_{1}}=\left\|u_{0}\right\|_{X_{\Omega}^{s, p, \theta}}$. Then, we define the complete metric space $\left(X_{1}, d_{1}\right)$ and the $\operatorname{map} \psi$ by

$$
\begin{aligned}
& X_{1}:=\left\{u \in L^{\theta}\left(0, \infty ; \dot{W}^{s, p}\left(\mathbb{R}^{3}\right)^{3}\right) \mid\|u\|_{Z_{1}} \leq 2\left\|u_{0}\right\|_{X_{\Omega}^{s, p, \theta}}\right\} \\
& d_{1}(u, v):=\|u-v\|_{Z_{1}} \\
& \psi(u)(t):=T_{\Omega}(t) u_{0}-N(u, u)(t)
\end{aligned}
$$

where $N(u, v)(t)$ is defined in (16). By the inequality (17), there exists a positive constant $C_{0}=C_{0}(\alpha, s, p, \theta)$ such that

$$
\begin{aligned}
\|\psi(u)\|_{Z_{1}} & \leq\left\|u_{0}\right\|_{X_{\Omega}^{s, p, \theta}}+C_{0}|\Omega|^{-\left\{1-\frac{1}{2 \alpha}\left(1+\frac{3}{p}-s\right)-\frac{1}{\theta}\right\}}\|u\|_{Z_{1}}^{2} \\
& \leq\left\|u_{0}\right\|_{X_{\Omega}^{s, p, \theta}}\left\{1+4 C_{0}|\Omega|^{-\left\{1-\frac{1}{2 \alpha}\left(1+\frac{3}{p}-s\right)-\frac{1}{\theta}\right\}}\left\|u_{0}\right\|_{\left.X_{\Omega}^{s, p, \theta}\right\}}\right.
\end{aligned}
$$


for all $u \in X_{1}$. Moreover, by using inequality (17), there exists a positive constant $C_{1}$ such that for $u, v \in X_{1}$,

$$
\begin{aligned}
\|\psi(u)-\psi(v)\|_{Z_{1}} & =\|N(u, u-v)+N(u-v, v)\|_{Z_{1}} \\
& \leq|\Omega|^{-\left\{1-\frac{1}{2 \alpha}\left(1+\frac{3}{p}-s\right)-\frac{1}{\theta}\right\}}\left(\|u\|_{Z_{1}}+\|v\|_{Z_{1}}\right)\|u-v\|_{Z_{1}} \\
& \leq 4 C_{1}|\Omega|^{-\left\{1-\frac{1}{2 \alpha}\left(1+\frac{3}{p}-s\right)-\frac{1}{\theta}\right\}}\left\|u_{0}\right\|_{X_{\Omega}^{s, p, \theta}}\|u-v\|_{Z_{1}} .
\end{aligned}
$$

Now, let us assume that initial velocity $u_{0} \in X_{\Omega}^{s, p, \theta}\left(\mathbb{R}^{3}\right)^{3} \cap \dot{H}^{s}\left(\mathbb{R}^{3}\right)^{3}$ satisfies

$$
\sup _{\Omega \in \mathbb{R} \backslash\{0\}}|\Omega|^{-\left\{1-\frac{1}{2 \alpha}\left(1+\frac{3}{p}-s\right)-\frac{1}{\theta}\right\}}\left\|u_{0}\right\|_{X_{\Omega}^{s, p, \theta}} \leq \min \left\{\frac{1}{8 C_{1}}, \frac{1}{4 C_{0}}\right\},
$$

we obtain from (4.1) and (4.2) that

$$
\|\psi(u)\|_{Z_{1}} \leq 2\left\|u_{0}\right\|_{X_{\Omega}^{s, p, \theta},} \quad\|\psi(u)-\psi(v)\|_{Z_{1}} \leq \frac{1}{2}\|u-v\|_{Z_{1}}
$$

for $u, v \in X_{1}$. Therefore, by the contraction mapping principle, there exists a unique solution $u \in X_{1}$ satisfying (3) for all $t>0$.

It remains to show that the solution $u \in X_{1}$ also belongs to $C\left([0, \infty) ; \dot{H}^{s}\left(\mathbb{R}^{3}\right)^{3}\right)$. Taking $\frac{1}{q}:=\frac{2}{p}-\frac{s}{3}$ with $1<q \leq 2$ and using Lemma 6 and Lemma 9,

$$
\begin{aligned}
\|u(t)\|_{\dot{H}^{s}} & \leq\left\|T_{\Omega}(t) u\right\|_{\dot{H}^{s}}+\|N(u, u)(t)\|_{\dot{H}^{s}} \\
& \leq C\left\|u_{0}\right\|_{\dot{H}^{s}}+C \int \frac{1}{(t-\tau)^{\left(\frac{3}{p \alpha}-\frac{s}{2 \alpha}-\frac{3}{4 \alpha}+\frac{1}{2 \alpha}\right)}}\|u \otimes v\|_{L^{p_{0}}} d \tau \\
& \leq C\left\|u_{0}\right\|_{\dot{H}^{s}}+C \int \frac{1}{(t-\tau)^{\left(\frac{3}{p \alpha}-\frac{s}{2 \alpha}-\frac{3}{4 \alpha}+\frac{1}{2 \alpha}\right)}}\|u\|_{\dot{W}^{s, q}}^{2} d \tau .
\end{aligned}
$$

By the Hölder inequality, we have

$$
\|u(t)\|_{\dot{H}^{s}} \leq C\left\|u_{0}\right\|_{\dot{H}^{s}}+C\left(\int_{0}^{t} \frac{1}{(t-\tau)^{\left(\frac{3}{p \alpha}-\frac{s}{2 \alpha}-\frac{3}{4 \alpha}+\frac{1}{2 \alpha}\right)\left(\frac{\theta}{2}\right)^{\prime}}} d \tau\right)^{\frac{1}{\left(\frac{\theta}{2}\right)^{\prime}}}\|u\|_{L^{\theta}\left(0, \infty ; \dot{W}^{s, p}\right)}^{2} .
$$

Since $\frac{1}{\theta}<\frac{1}{2}+\frac{1}{8 \alpha}+\frac{s}{4 \alpha}-\frac{3}{2 \alpha p}$, the time integral on the right hand side of (22) converges and

$$
\left(\int_{0}^{t} \frac{1}{(t-\tau)^{\left(\frac{3}{p \alpha}-\frac{s}{2 \alpha}-\frac{3}{4 \alpha}+\frac{1}{2 \alpha}\right)\left(\frac{\theta}{2}\right)^{\prime}}} d \tau\right)^{\frac{1}{\left(\frac{\theta}{2}\right)^{\prime}}}=C t^{2\left(\frac{1}{2}+\frac{1}{8 \alpha}+\frac{s}{4 \alpha}-\frac{3}{2 \alpha p}-\frac{1}{\theta}\right)} .
$$

Which implies that $u(t)$ belongs to $\left.\dot{H}^{s}\left(\mathbb{R}^{3}\right)^{3}\right)$ for all $t \geq 0$. Similarly, we see that $u \in$ $C\left([0, \infty) ; \dot{H}^{s}\left(\mathbb{R}^{3}\right)^{3}\right)$. This completes the proof of Theorem 1 .

Proof of Theorem 2. It is not difficult to check that the indices $\alpha, s$ and $p$ given in the Theorem 2 satisfy the assumptions of Lemma 6 and Lemma 11. Suppose $u_{0} \in X_{\Omega}^{p}\left(\mathbb{R}^{3}\right)^{3} \cap$ $\dot{H}^{s}\left(\mathbb{R}^{3}\right)^{3}$ satisfies $\operatorname{div} u_{0}=0$. By Lemma 6 and the definitions of $\|\cdot\|_{Y_{2}}$ and $\|\cdot\|_{Z_{2}}$, we see that there exists a positive constant $C_{0}=C_{0}(s)$ such that $\left\|T_{\Omega}(\cdot) u_{0}\right\| Y_{2} \leq C_{0}\left\|u_{0}\right\|_{\dot{H}^{s}}$ and $\left\|T_{\Omega}(\cdot) u_{0}\right\|_{Z_{2}}=\left\|u_{0}\right\|_{X_{\Omega}^{p}}$. Then, we define the complete metric space $\left(X_{2}, d_{2}\right)$ and the $\operatorname{map} \psi$ by

$$
\begin{aligned}
& X_{2}:=\left\{u \in B C\left([0, \infty) ; \dot{H}^{s}\left(\mathbb{R}^{3}\right)^{3}\right) \mid\|u\|_{Z_{1}} \leq 2\left\|u_{0}\right\|_{X_{\Omega}^{s, p, \theta}}\right\} \\
& d_{2}(u, v):=\|u-v\|_{\gamma_{2}} \\
& \psi(u)(t):=T_{\Omega}(t) u_{0}-N(u, u)(t),
\end{aligned}
$$


where $N(\cdot, \cdot)(t)$ is defined in (16). By the inequality (18), there exists a positive constant $C_{1}=C_{1}(\alpha, s, p)$ such that

$$
\begin{aligned}
\|\psi(u)\|_{Y_{2}} & \leq C_{0}\left\|u_{0}\right\|_{H^{s}}+C_{1}\|u\|_{Y_{2}}\|u\|_{Z_{2}} \\
& \leq C_{0}\left\|u_{0}\right\|_{\dot{H}^{s}}\left\{1+4 C_{1}\left\|u_{0}\right\|_{X_{\Omega}^{p}}\right\}
\end{aligned}
$$

for all $u \in X_{2}$. Similarly, by the inequality (19), there exists a positive constant $C_{2}=$ $C_{2}(\alpha, p)$ such that

$$
\begin{aligned}
\|\psi(u)\|_{Z_{2}} & \leq\left\|u_{0}\right\|_{X_{\Omega}^{p}}+C_{2}\|u\|_{Z_{2}}\|u\|_{Z_{2}} \\
& \leq\left\|u_{0}\right\|_{X_{\Omega}^{p}}\left\{1+4 C_{2}\left\|u_{0}\right\|_{X_{\Omega}^{p}}\right\}
\end{aligned}
$$

for all $u \in X_{2}$. Moreover, by using Lemma 11, there exists a positive constant $C_{3}=$ $C_{3}(\alpha, s, p)$ such that for $u, v \in X_{2}$,

$$
\begin{aligned}
\|\psi(u)-\psi(v)\|_{Y_{2}} & =\|N(u, u-v)+N(u-v, v)\|_{Y_{2}} \\
& \leq C_{3}\left(\|u\|_{Z_{2}}+\|v\|_{Z_{2}}\right)\|u-v\|_{Y_{2}} \\
& \leq 4 C_{3}\left\|u_{0}\right\|_{X_{\Omega}^{p}}\|u-v\|_{Y_{2}} .
\end{aligned}
$$

Now, let us assume that initial velocity $u_{0} \in X_{\Omega}^{p}\left(\mathbb{R}^{3}\right)^{3} \cap \dot{H}^{s}\left(\mathbb{R}^{3}\right)^{3}$ satisfies

$$
\sup _{\Omega \in \mathbb{R}} \leq \min \left\{\frac{1}{8 C_{3}}, \frac{1}{4 C_{1}}, \frac{1}{4 C_{2}}\right\}
$$

we obtain from (4.4), (4.5) and (4.6) that

$$
\|\psi(u)\|_{Y_{2}} \leq 2 C_{0}\left\|u_{0}\right\|_{\dot{H}^{s}}, \quad\|\psi(u)\|_{Z_{2}} \leq 2\left\|u_{0}\right\|_{X_{\Omega}^{p}}, \quad\|\psi(u)-\psi(v)\|_{Y_{2}} \leq \frac{1}{2}\|u-v\|_{Y_{2}}
$$

for $u, v \in X_{2}$. Therefore, by the contraction mapping principle, there exists a unique solution $u \in X_{2}$ satisfying (3) for all $t>0$. This completes the proof of Theorem 2.

Author Contributions: Writing—original draft preparation, Jia L.; writing—review and editing, Xiaochun.S.; supervision, Jihong.Z.; project administration, Xiaochun.S.; funding acquisition, Xiaochun.S. All authors have read and agreed to the published version of the manuscript.

Funding: This research was funded by National Natural Science Foundation of China grant number 11601434.

Institutional Review Board Statement: : Not applicable.

Informed Consent Statement: : Not applicable.

Acknowledgments: The authors thank the referees for many valuable comments and suggestions.These greatly improved the presentation of our results.

Conflicts of Interest: The authors declare no conflict of interest. The funders had no role in the design of the study; in the collection, analyses, or interpretation of data; in the writing of the manuscript, or in the decision to publish the results.

\section{References}

1. Babin, A., Mahalov, A. and Nicolaenko, B. Regularity and integrability of 3D Euler and Navier-Stokes equations for rotating fluids. Asymptot. Anal. 1997, 15, 103-150.

2. Babin, A., Mahalov, A. and Nicolaenko, B. Global regularity of the 3D Rotating Navier-Stokes equations for resonant domains. Indiana Univ. Math. J. 1999, 48, 1133-1176.

3. Babin, A., Mahalov, A. and Nicolaenko, B. 3D Navier-Stokes and Euler equations with initial data characterized by uniformly large vorticity. Indiana Univ. Math. J. 2001, 50, 1-35. 
4. Bahouri, H., Chemin,J.-Y. and Danchin, R. In Fourier analysis and nonlinear partial differential equations, Grundlehren der Mathematischen Wissenschaften, vol. 343, Springer-Verlag, Berlin, Heidelberg, 2011;

5. Chemin, J.-Y., Desjardin, B., Gallagher, I. and and Grenier, E. Anisotropy and dispersion in rotating fluids, In Nonlinear PDEs and Applications, Stud. Math. Appl. 31, North Holland, 2002;

6. Chemin, J.-Y., Desjardin, B., Gallagher, I. and and Grenier, E. In Mathematical geophysics. An introduction to rotating fluids and the Navier-Stokes equations, Volume 32 of Oxford Lecture Series in Mathematics and its Applications. The Clarendon Press, Oxford University Press, Oxford, 2006;

7. Xiaochun, S. and Yong, D. Dispersive effect of the Coriolis force and the local well-posedness for the Navier-Stokes-Coriolis system. J. Evol. Equ. 2020, 20, 335-354.

8. Yong, D. and Xiaochun, S. Strichartz estimates for parabolic equations with higher order differential operator. Sci. China Math. 2015, 58, 1047-1062.

9. Fujita, H. and Kato, T.On the Navier-Stokes initial value problem I. Arch. Ration. Mech. Anal. 1964, 16, $269-315$.

10. Giga, Y., Inui, K., Mahalov, A. and Saal, J. Uniform global solvability of the rotating Navier-Stokes equations for nondecaying initial data. Indiana Univ. Math. J. 2008, 57, 2775-2791.

11. Hieber, M. and Shibata, Y. The Fujita-Kato approach to the Navier-Stokes equations in the rotational. Math. Z. 2010, 265, 2481-491.

12. Iwabuchi, T. and Takada, R. Global solutions for the Navier-Stokes equations in the rotational framework. Math. Ann. 2013, 357, 727-741.

13. Iwabuchi, T. and Takada, R. Global well-posedness and ill-posedness for the Navier-Stokes equations with the Coriolis force in function spaces of Besov type. J. Funct. Anal. 2014, 267, 1321-1337.

14. Iwabuchi, T. and Takada, R. Dispersive effect of the Coriolis force and the local well-posedness for the Navier-Stokes equations in rotational framework. Funkcial. Ekvac. 2015, 58, 365-385.

15. Ohyama, H. Global well-posedness for the Navier-Stokes equations with the Coriolis force in function spaces characterized by semigroups. J. Math. Fluid Mech. 2021, 23, 1-10.

16. Kato, T. Strong $L^{p}$-solutions of the Navier-Stokes equation in $\mathbb{R}^{m}$, with applications to weak solutions. Math. Z. 1984, 187, 471-480.

17. Koch, H. and Tataru, D. Well-posedness for the Navier-Stokes equations. Adv. Math. 2001, 157, 22-35.

18. Koh, Y., Lee, S. and Takada, R. Dispersive estimates for the Navier-Stokes equations in the rotational framework. Adv. Differential Equations 2014, 19, 857-878.

19. Konieczny, P. and Yoneda, Y. On dispersive effect of the Coriolis force for the stationary Navier-Stokes equations. J. Differential Equations 2011, 250, 3859-3873.

20. Kozono, H., Ogawa, T. and Taniuchi, Y. Navier-Stokes equations in the Besov space near $L^{\infty}$ and BMO. Kyushu J. Math. 2003, 57, 303-324.

21. Lions, J. L. In Quelques méthodes de résolution des problémes aux limites non linéaires. Dunod; Gauthier-Villars, Paris, 1969;

22. Changxin, M., Baoquan, Y. and Bo, Z. Well-posedness of the Cauchy problem for the fractional power dissipative equations. Nonlinear Anal. 2008, 68, 461-484.

23. Stein, E. M. In Singular integrals and differentiability properties of functions. Princeton University Press, Princeton, 1970;

24. Jiahong, W. Generalized MHD equations. J. Differential Equations 2003, 195, 284-312.

25. Jiahong, W. The generalized incompressible Navier-Stokes equations in Besov spaces. Dyn. Partial Differ. Equ. 2004, 4, 381-400.

26. Jiahong, W. Lower bounds for an integral involving fractional Laplacians and the generalized Navier-Stokes equations in Besove spaces. Commun. Math. Phys. 2006, 263, 803-831.

27. Zhichun, Z. Well-posedness for fractional Navier-Stokes equations in the critical spaces close to $\dot{B}_{\infty, \infty}^{-(2 \beta)-1}\left(\mathbb{R}^{n}\right)$. Dyn. Partial Differ Equ. 2010, 7, 25-44. 